Short Communication

\title{
CHAOS SCIENCE \& THE PARADOX IN ELECTRO CARDIOGRAPHIC INTERPRETATIONS
}

\author{
Arunachalam Kumar \\ Professor of Anatomy, K.S. Hegde M edical Academy \\ Nitte University, Mangalore - 575 018, India \\ E-mail : dean.kshema@nitte.edu.in
}

\begin{abstract}
:
The application of the radically and new non-linear science of 'chaos' has been slow in acceptance in the medical field; each and every diagnostic, investigative and pharmaco-therapeutic process involved in health science is dictated by direct Newtonian tenets of causeeffect and linear mathematical derivations. Exclusive and total dependence on scalar models have compartmentalized patient profiles into rigid cocoons such as age, sex, weight, height, physiological and biochemical evaluations making interventional therapeutic regimens solely dictated by our over-riding choice to use scales of convenience for every single one of our evaluation, assessment and treatment criteria.

The paradoxical and skewed results in seemingly normal processes, when viewed through 'chaos' systems, show how wrong our total reliance on scale and linear applications are! As an example of how terribly paradoxical inferences can be, I have electrocardiography, a common and widely used diagnostic process
\end{abstract}

\section{Introduction:}

The new field of theoretical biology, spawned by the advent and ready acceptance of the 'chaos' model has lead to an explosion in nascent schools of thought that challenge hitherto entrenched linear mathematical Newtonian and Euclidian principles in natural sciences ${ }^{1}$. Fractal configurations and such other non-linear systems have made the need for a second look at physiodynamic processes of the human biology, mandatory. Despite rapid strides in the alternate chaotic and randomness applications, the world of medicine is still loathe to modify it's dependence and confidence in pharmacokinetic therapeutic solutions based on cause-effect and Euclidean transpolation linear systems, both in diagnosis and treatment. This paper very briefly, illustrates the fallacy in such approaches, using the record of the electrophysiological function of the heart (ECG) as an example. The paradox in ECG interpretation as clue to functional status of the heart, is demonstrated here when the principles of 'chaos' are applied as diagnostic tool.

\section{Materials \& Methods :}

Electrocardiographic recordings of three adult, normal males, applying Lead II (bipolar) were made. Twenty contractions of the heart and its record were studied from each volunteer. The ventricular depolarisation wave, QR, was isolated from the PQRST complex from every cardiac cycle. The QR spike was next projected onto a giant wallmounted screen to magnification of 100 ( $1 \mathrm{~cm}$ to 1 meter). The projected on-screen spike height was then measured to the nearest $\mathrm{mm}$. The values were tabulated and analyzed.

\section{Observations:}

When measured in its projected magnified form, each and every QR spike of a typical PQRST segment of the ECG record had its own individual and distinct height; the heights varied randomly and erratically even in tandem recordings within a single record-strip of the same individual. No two heights were identical. The characteristic, randomness and erraticity of variations in heights appeared to be obvious features in any ECG taken in same lead in normal individuals (Fig I, II \& III).

\section{Discussion :}

Electrocardiography, is based on Einthoven's principles, quantify the electrical activity of the heart. The recordings of electrical discharges are from the nodal pacemaker of cardiac tissue are made through different leads. The millivoltages of the depolarisation process of the generation and conduction of electrical impulses form the characteristic PQRST segments of a typical ECG. In observation made from this study, the seemingly regular 

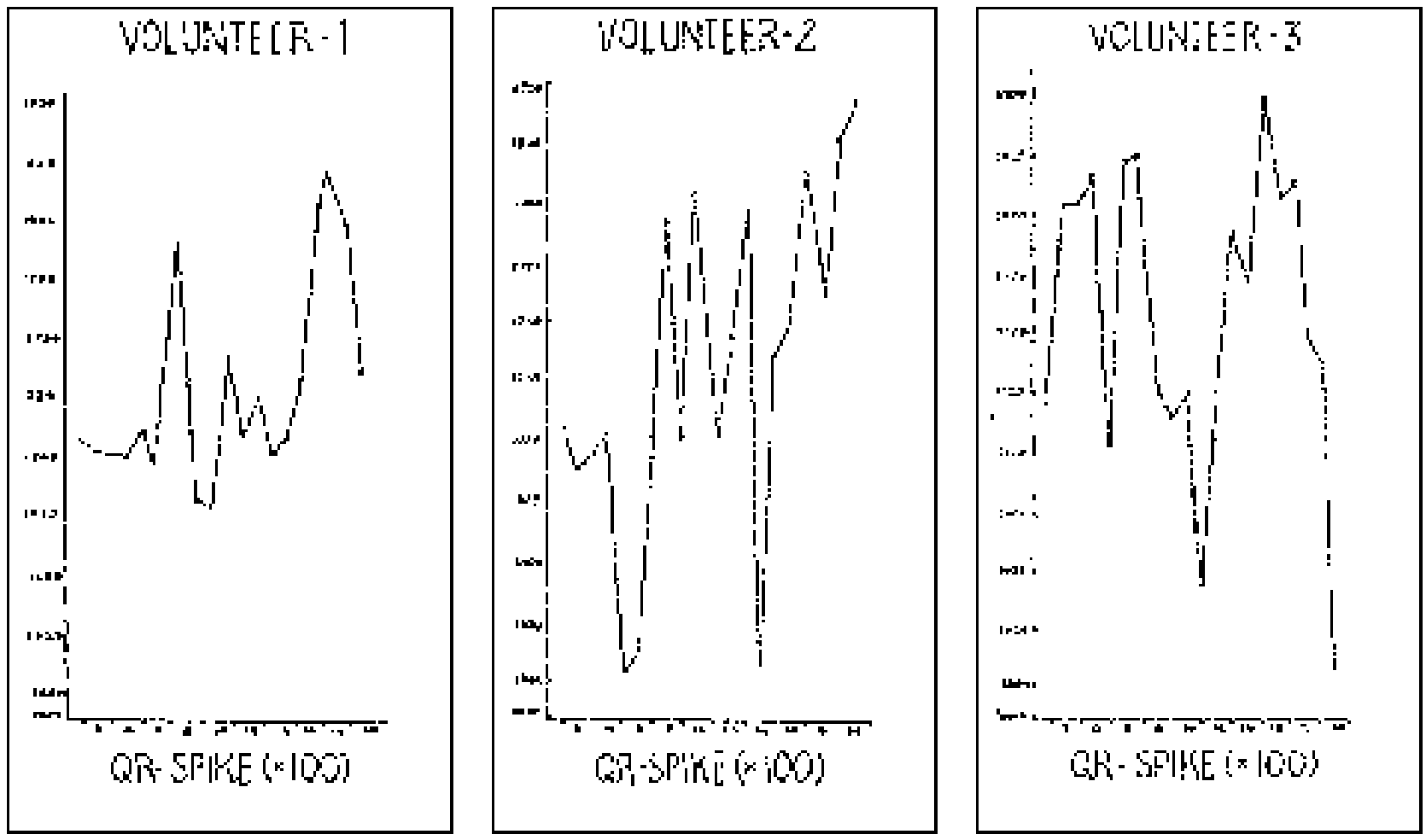

millivolt strengths recorded as ECGs in normal individuals, are apparently if altered in scale or magnified.

The wide fluctuations in spike heights observed at 100 times scale, demonstrates the absolute randomness in the electrical activity of the heart even within a single cardiac cycle. The chaotic behavior, according to non-linear chaos theory, isnormal and signifies good health.

The magnified spike measures clearly show that our long held historic view of the order and pattern of electrocardiographs and their interpretations could well be based on assumptions and presumptions that may well be fallacious. Our dependence on convenience of scale adopted by the ECG instrumentation processes, rather than accuracy and correctness, are dictated primarily by ease of facility and simplicity of its usage.

The science of chaos states that irregularity denotes wellbeing of physiological functions. Regularity forebodes ill health. Randomness and unpredictability are natural and innately normal processes: Disturbances and disruptions in erratic activity and behaviors patterns leads to disease and dysfunction.
The simple rhythms and conventional periodic patterns induced by phase mode locking in physiological activities in man, maybe is not be as sacrosanct as held till now. Every function has inherent randomness directly and increasingly proportional to robustness. The oscillation of Poincare (butterfly) graph between health and ill health attractors are said to show regularity in records as outcome of the diseased states. Indeed, by analogy, the ECGs should be 'most' regular in only when static, as in death - a fact well demonstrated in the absolute flat ECG record of the dead human heart. By interpolation malfunctioning heart, fibrillating or fluttering should produce ECG records, that when magnified, show less erraticity in the entire PQRST.

Though we have, for purpose of demonstration of nonlinear chaotic system application, chosen only a single lead, part of the ECG wave and strip and used only three volunteers with normal hearts, it is our view that, even from the limited data studied, it is obvious that inherent randomness indeed is characteristic in normal cycles of the cardiovascular system and that electrical discharge from the pacemaker is as a norm, fluctuant and erratic ${ }^{2}$.

Medicine, both diagnostic, clinical and treatment both pharmacological and therapeutic, could well reassess it's 
stress on normal ranges and standardization's, averages and means and statistical reliance on convenience derived

\section{References:}

1. Arunachalam Kumar, Hegde B.M \& \& Prabhu .S, 1996, The chaos theory, cardiology \& electrocardiographs; Proceedings of the National Conference on Chaos Theory, Bangalore

2. Rao A.V \& Arunachalam Kumar, Linearity or chaos? An parameters and instrumentations such as in electrocardiography.

electrocardiographic perspective, 1998 XIX National; Conference of Indian Association of Biomedical Scientists, M angalore

3. Rao A.V \& Arunachalam Kumar, 1998, Intrinsic cardiac randomness \& diagnostic pitfalls, Proceedings of the National Conference on Biomedical Engineering, Ed. Niranjan U.C., M anipal 